\title{
Current systems of coronal loops in 3D MHD simulations
}

\author{
J. Warnecke ${ }^{1}$, F. Chen ${ }^{2,1}$, S. Bingert ${ }^{3}$, and H. Peter ${ }^{1}$ \\ 1 Max-Planck-Institut für Sonnensystemforschung, Justus-von-Liebig-Weg 3, 37077 Göttingen, Germany \\ e-mail: warnecke@mps.mpg.de \\ 2 High Altitude Observatory, National Center for Atmospheric Research, 3080 Center Green Dr., Boulder, CO 80301, USA \\ 3 Gesellschaft für wissenschaftliche Datenverarbeitung mbH Göttingen, Am Faßberg 11, 37077 Göttingen, Germany
}

Received 18 November 2016 / Accepted 19 May 2017

\begin{abstract}
Aims. We study the magnetic field and current structure associated with a coronal loop. Through this we investigate to what extent the assumptions of a force-free magnetic field break down and where they might be justified.

Methods. We analyze a three-dimensional (3D) magnetohydrodynamic (MHD) model of the solar corona in an emerging active region with the focus on the structure of the forming coronal loops. The lower boundary of this simulation is taken from a model of an emerging active region. As a consequence of the emerging magnetic flux and the horizontal motions at the surface a coronal loop forms self-consistently. We investigate the current density along magnetic field lines inside (and outside) this loop and study the magnetic and plasma properties in and around this loop. The loop is defined as the bundle of field lines that coincides with enhanced emission in extreme UV.

Results. We find that the total current along the emerging loop changes its sign from being antiparallel to parallel to the magnetic field. This is caused by the inclination of the loop together with the footpoint motion. Around the loop, the currents form a complex non-force-free helical structure. This is directly related to a bipolar current structure at the loop footpoints at the base of the corona and a local reduction of the background magnetic field (i.e., outside the loop) caused by the plasma flow into and along the loop. Furthermore, the locally reduced magnetic pressure in the loop allows the loop to sustain a higher density, which is crucial for the emission in extreme UV. The action of the flow on the magnetic field hosting the loop turns out to also be responsible for the observed squashing of the loop.

Conclusions. The complex magnetic field and current system surrounding it can only be modeled in 3D MHD models where the magnetic field has to balance the plasma pressure. A one-dimensional coronal loop model or a force-free extrapolation cannot capture the current system and the complex interaction of the plasma and the magnetic field in the coronal loop, despite the fact that the loop is under low- $\beta$ conditions.
\end{abstract}

Key words. magnetohydrodynamics (MHD) - Sun: corona - Sun: magnetic fields

\section{Introduction}

The solar corona is characterized by its high temperature and low plasma density. Mostly, there the magnetic field dominates the structures and dynamics of the coronal plasma as quantified by a low value of plasma- $\beta$. This is the ratio of magnetic to gas pressure. Under the assumption of a low- $\beta$ corona, the magnetic field can be modeled using force-free extrapolation of the photospheric magnetic field (for a review, we refer to Wiegelmann 2008). In these models, the force-freeness is ensured by requiring the currents to be parallel or antiparallel to the magnetic field, which turns out to be not always valid (Peter et al. 2015).

Since the coronal model of Gudiksen \& Nordlund (2002, 2005) three-dimensional (3D) magnetohydrodynamics (MHD) models can produce a loop-dominated corona in a realistic setup. In particular these models generate self-consistently high coronal temperatures through the braiding of magnetic field lines (Parker 1972) driven by photospheric motions of the magnetic footpoints (e.g., Bingert \& Peter 2011). Such models produce a distribution of energy input consistent with the nanoflare model (Bingert \& Peter 2013). The coronal emission synthesized from these models fits well with observed coronal loop properties. This applies to the spectroscopic properties, for example, average Doppler shifts (Peter et al. 2004, 2006; Hansteen et al. 2010), the distribution of the emission within an active region or along a loop (e.g., Mok et al. 2005, 2008), or the appearance of (small) loops based on the observed photospheric magnetic field (Bourdin et al. 2013). Therefore these models can be considered as a good representation of coronal structures and dynamics on the resolved scale (e.g., Peter 2015). The magnetic field in these simulations turns out to be close to force-free. This is indicated by the exponential drop of both the averaged heating rate $\left(\propto j^{2}\right)$ and magnetic energy $\left(\propto B^{2}\right)$ with the same scale height (Gudiksen \& Nordlund 2005, here $j$ and $B$ are the current density and the magnetic field strength, respectively). More direct support for the force-freeness is through the small angle between the current and the magnetic field, which is on average smaller than $20^{\circ}$ in a stable active region in the model of Bingert \& Peter (2011), shown in Bingert (2009). However, a force-free magnetic field can also be achieved by a simplified high plasma- $\beta$ coronal model (Warnecke \& Brandenburg 2010).

The most crucial test to ensure the field is force-free is to check the angle of the magnetic field to the electric currents, as well as the total current along the loop. While the angle should be close to zero or $180^{\circ}$, of course, furthermore the absolute value of the current should be approximately constant. Because the magnetic field is expanding with height, this latter criterium implies that the total current through the cross section of a flux tube hosting the loop defined by a set of field lines has to be 

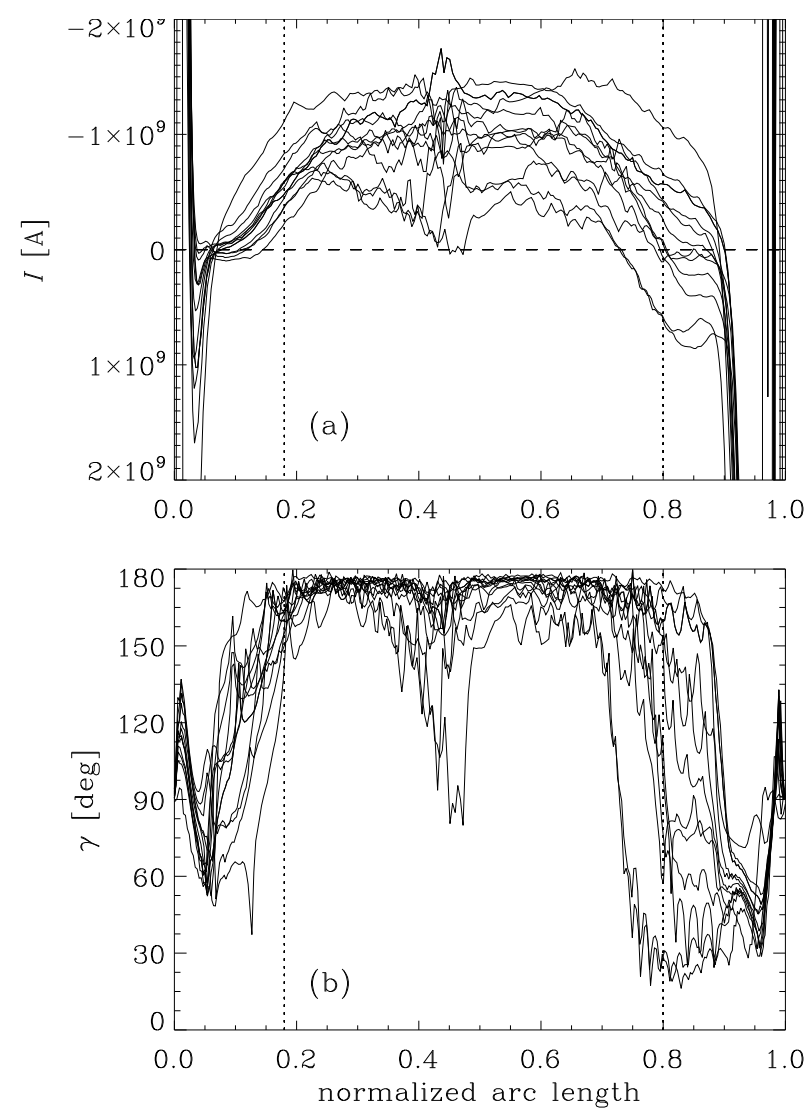

Fig. 1. Testing the force-freeness in a 3D MHD model of a stable active region. Panel a: total current, $I$, along the magnetic field, $\boldsymbol{B}$, where $I$ is integrated over the cross-section of the expanding flux tube. Panel $b$ : displays the angle $\gamma$ between the current density, $\boldsymbol{J}$, and $\boldsymbol{B}$. Both $I$ and $\gamma$ are plotted for twelve selected field lines in an EUV loop over the normalized arc length (i.e., 0 and 1 are in the photosphere and the apex is at about 0.5). $I$ and $\gamma$ are defined in Eqs. (1) and (2), respectively. The hot coronal part of the loop (with temperatures above $1 \mathrm{MK}$ ) is found between the vertical dotted lines. Based on data from Bingert \& Peter (2011). See Sect. 1

constant along the loop. However, the crucial part is that the current should not change sign. We analyze this briefly for the model of a stable active region from Bingert \& Peter (2011) in which a quasi-steady loop forms and exists for about $30 \mathrm{~min}$, similar to an observed loop. We display the total current and the angle in Fig. 1 for twelve selected field lines within the loops seen in extreme UV emission (EUV). From Fig. 1a it is clear that indeed the total current is more or less constant in the coronal part. Of course, this is no longer the case in the lower chromospheric and photospheric part of the loop. Furthermore, the sign of the current, and therefore its direction, is mostly positive in the coronal region. This behavior is underlined by the distribution of the angle $\gamma$ between the magnetic field and the current density, as shown in Fig. 1b. In the coronal region, $\gamma$ is preferential close to $180^{\circ}$, indicating an antiparallel current along most of the magnetic field lines. From this we conclude that the magnetic field in the hot dense loops of this model is indeed force-free. The (rough) alignment of the currents with the magnetic field is also found in models with a simpler magnetic geometry, for example, in the MHD model of Galsgaard \& Nordlund (1996) of a loop in a straight geometry. In their reduced MHD model Rappazzo et al. (2008) investigated what role MHD turbulence plays in such a setup for the braiding of the magnetic field.
The question arises of whether or not a more dynamic model of an active region with a changing magnetic field in the corona would still host a magnetic field in a nearly force-free state. This motivates the analysis of a 3D MHD model of an emerging active region, and for this purpose we employ the model by Chen et al. $(2014,2015)$, who simulated the coronal response to the formation of an active region through flux emergence in the photosphere. There, a coronal magnetic loop structure forms resulting in a bright loop seen in EUV at coronal temperatures. While this is a model of an emerging region, a real emerging active region on the Sun will be much more violent, and thus our considerations for violations of the force-free state should be considered as a lower limit. Where we see significant deviation from a forcefree magnetic field in this model, for the real Sun, much more severe violations should be expected.

A further motivation for our study are the recently performed plasma laboratory experiments of a coronal loop eruption (Oz et al. 2011; Myers et al. 2015). In their setup, a hot plasma loop, considered to be the equivalent of a coronal loop, is generated by a constant current through a plasma confined by a complex loop-like magnetic field structure. This leads to a strong Lorentz-force, which is partly balanced by their plasma and in consequence causes an eruption. In their setup the magnetic field turns out to be mostly non-force-free. Even though these experiments are still far from the configuration of the solar corona they provide an interesting path towards understanding the eruption mechanism. Our study might provide some new insight into the current systems and magnetic field in and around coronal loops and therefore contribute to the improvement of the interpretation and further development of the experiments.

While the laboratory aspect has its own appeal, the main interest of our study is to test to what extent a changing magnetic field in an emerging active region is in a force-free state. For this we investigate data from an existing 3D MHD model (Sect. 2), investigate the current systems around the loop forming in this model (Sect. 3.1) and discuss its connection with the magnetic field structure (Sect. 3.3). Then we show how the plasma properties can influence the magnetic field structure and evolution (Sects. 3.4 and 3.5). This leads us to the limitations of the forcefree assumptions for emerging active regions in Sect. 4.

\section{Three-dimensional magnetohydrodynamics model of the active region corona}

In this work, we analyze data from the model described in detail in Chen et al. (2014, 2015). The modeling strategy of that model is based on Bingert \& Peter (2011, 2013), but all data shown here are from Chen et al. $(2014,2015)$. In short, in these $3 \mathrm{D}$ simulations the corona is modeled by solving the equations of compressible MHD with the PENCIL CODE ${ }^{1}$ including the induction equation, continuity equation, momentum equation, and energy equation. Bingert \& Peter $(2011,2013)$ used the photospheric magnetogram from an observed well evolved active region as the lower boundary of the magnetic field that is driven by horizontal motions. This leads to a more or less stable active region corona. In contrast, Chen et al. $(2014,2015)$ use the significantly changing magnetic field (and velocity field) in the photosphere from the flux emergence simulation of Rempel \& Cheung (2014). This flux emergence simulation is less violent than the emergence model by Cheung et al. (2010) but still features the transition from basically no magnetic field at the photosphere (our lower boundary) to two strong opposite polarity sunspots.

1 http://github.com/pencil-code 

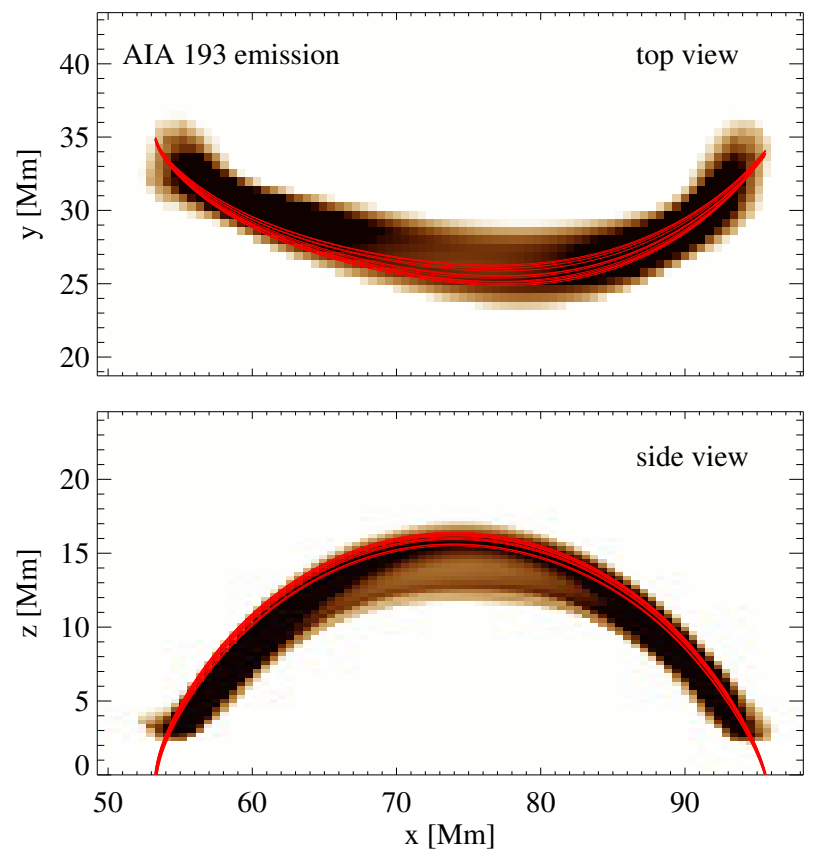

Fig. 2. Snapshot of coronal loop and field lines studied here. The background image shows the synthesized EUV emission as it would be seen in the $193 \AA$ A channel of AIA showing mainly $1.5 \mathrm{MK}$ hot plasma. Overplotted are twelve selected magnetic field lines (red) within the EUV loop. This shows a snapshot at time $t=12.5 \mathrm{~min}$ based on the data from the model by Chen et al. $(2014,2015)$. The two panels show a view from straight above (top panel) and the side (bottom panel) of part of the computational domain; we refer to Sect. 2.

In the coronal model of Chen et al. $(2014,2015)$ coupled to the flux emergence simulation this results in the formation of a coronal loop structure that is non-steady in nature. Thus this coronal simulation is well suited to studying the currents hosted in an active-region corona during phases of significant evolution.

We focus our study mainly on a snapshot at $t=12.5 \mathrm{~min}$ of the coronal simulation of Chen et al. $(2014,2015)^{2}$. Besides twodimensional (2D) cuts and 3D volume rendering, we follow individual field lines and determine physical properties along them using the same technique as in Bingert \& Peter $(2011,2013)$ and Chen et al. (2014). As an illustration, we show in Fig. 2 the field lines over-plotted with the bright loop visible in EUV emission at time $t=12.5 \mathrm{~min}$. Here we display the emission as it would be seen by the Atmospheric Imaging Assembly (Boerner et al. 2012) in its $193 \AA$ channel showing basically Fe XII forming at about $1.5 \mathrm{MK}$. All the field lines are well inside the EUV loop and are therefore well suited to describing its plasma, magnetic field, and current properties. The EUV loop is bright only above $\approx 3 \mathrm{Mm}$ because of the sensitivity in terms of temperature of the AIA $193 \AA$ channel. The currents along these field lines in the loop play a crucial role for the heating of the loop and its visibility in the EUV (for further details see Chen et al. 2014).

\section{Results}

\subsection{Electric current system in and around the loop}

We start by analyzing currents along the magnetic field lines in the loop structure seen in EUV. For this we define the total

\footnotetext{
2 Actually this is more than an hour from the start of the coronal simulation when the first clear loop has formed. For the definition of the zero-time we refer to Chen et al. $(2014,2015)$ and their online material.
}
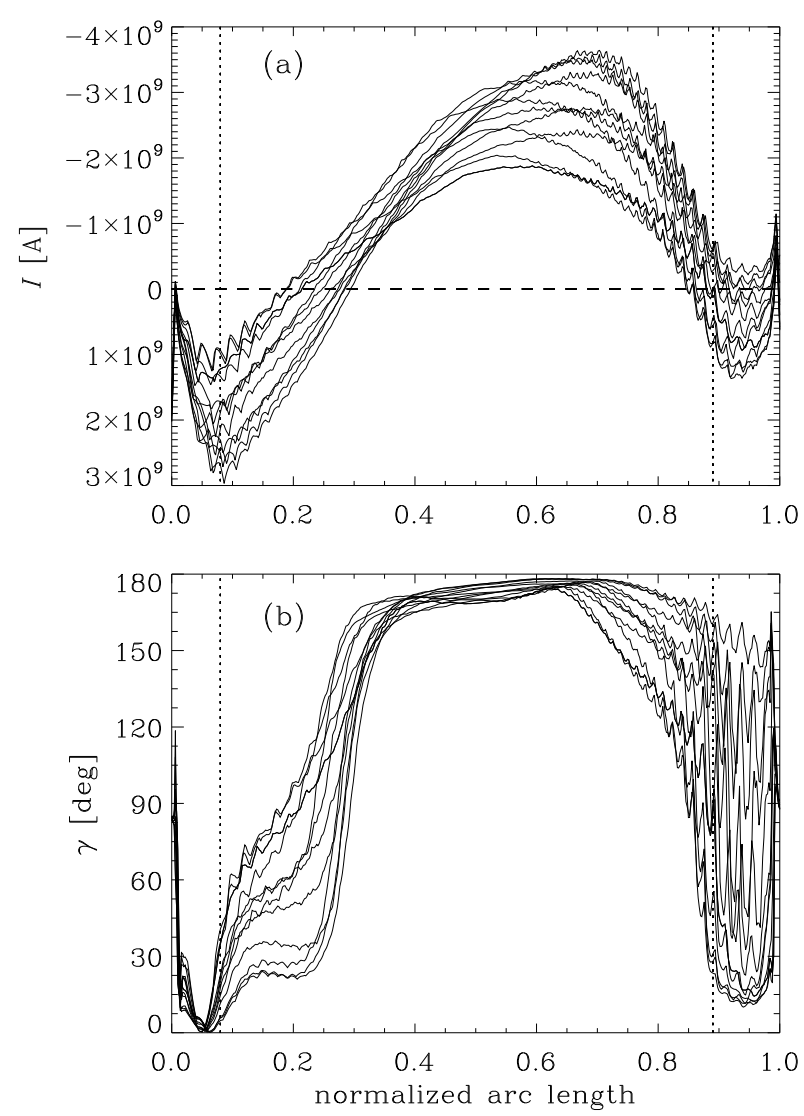

Fig. 3. Current properties along a loop in an emerging active region of Chen et al. (2014, 2015). The format is the same as for the loop in the stable active region in Fig. 1. Panel a shows the total current $I$ along the loop that now changes sign and panel $b$ displays the angle $\gamma$ between current and magnetic field. Just as in Fig. 1 we show $I$ and $\gamma$ for twelve selected field lines in the loop (cf. Fig. 2) over the normalized arc length. Zero arc length is at the left footpoint of the loop (see Fig. 4). The two vertical dotted lines indicate the coronal region (above $1 \mathrm{MK}$ ) and the dashed line indicates zero current. We refer to Sect. 3.1.

current $I$ along the magnetic field $\boldsymbol{B}$ as

$I=\frac{\boldsymbol{J} \cdot \boldsymbol{B}}{|\boldsymbol{B}|} \mathcal{A}, \quad$ with $\quad \mathcal{A}=\frac{\left|B_{z}(z=0)\right|}{|\boldsymbol{B}|} \pi r_{0}^{2}$,

where $\boldsymbol{J}=\mu_{0}^{-1} \nabla \times \boldsymbol{B}$ is the current density and $\mathcal{A}$ is the crosssection of the loop with the constant $r_{0}=0.5 \mathrm{Mm}$. By following an individual field line and normalizing by the magnetic field this is equivalent to following a flux tube that has an infinitesimally small diameter at the lower boundary. Just for convenience we used a value of $2 r_{0}=1 \mathrm{Mm}$ at the height $z=0$, that is, the bottom boundary in the photosphere. The loop is then expanding according to flux conservation, that is, the cross-section $\mathcal{A}$ increases with decreasing magnetic field strength $|\boldsymbol{B}|$. Basically calculating $I$ through Eq. (1) corresponds to integrating the current density over the cross-section of a magnetic flux tube defined by a collection of field lines. The actual choice of $r_{0}$ does not matter in our analysis and is only used for convenience to get sensible units and values for $I$. A different value of $r_{0}$ would only result in a different amplitude of the $I$ but would not give a different dependency along the loop.

We now address the question of if and how the total current changes along the loop in this emerging active region model. For this we plot in Fig. 3a the total current $I$ as a function of normalized arc lengths for twelve different field lines, where the 


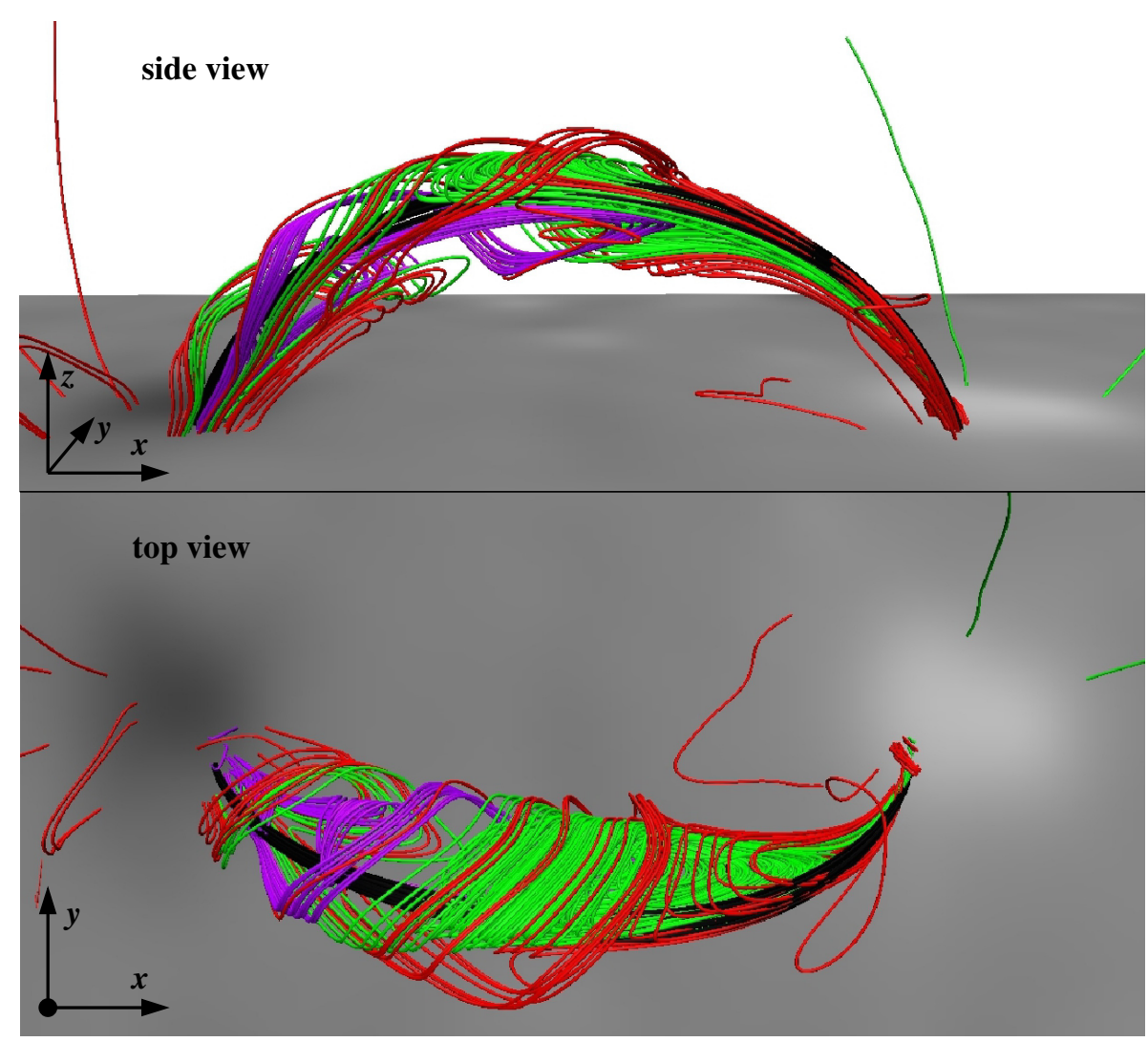

Fig. 4. 3D rendering of the current and magnetic field lines of the loop in the emerging active region of Chen et al. $(2014,2015)$. The two panels show the side view (top panel) and the top view (bottom panel) on part of the computational box. The black lines are the field lines within the EUV loop (same as in Fig. 2) and the colored lines show current lines (or streamlines of the vector field of the current). The gray scale images show the vertical magnetic field at a height of $z=2.9 \mathrm{Mm}$ at the base of the corona where the field already expanded and is rather smooth. The three color sets of current lines traced from different starting points: near the positive polarity (red), negative polarity (purple), and from the loop apex (green). We refer to Sect. 3.1. coronal part is found between the vertical dotted lines. In this coronal part, the plasma on the respective field line is hotter than $1 \mathrm{MK}$. All the field lines show a roughly similar behavior: In about three quarters of the coronal part the total current $I$ is negative, whereas in the remaining quarter $I$ is positive. This flipping in sign is also consistent with the angle $\gamma$ between magnetic field and currents, which changes from almost $180^{\circ}$ to $30^{\circ}$ at the same location (Fig. 3b). At first glance this sign flip of the currents seems to be counter-intuitive because it is not consistent with the approximation of a force-free magnetic field, where the total current has to be constant and either parallel or antiparallel throughout the whole loop (cf. Fig. 1a and Sect. 1). Thus in this simulation certainly the magnetic field is not force-free. Of course, we have to investigate why the magnetic field and current density are parallel on one side of the loop and antiparallel on the other side.

As a first step, we track the field lines or streamlines of the vector field of the current density. In the following we refer to these as current lines; they basically track the currents in our system. In Fig. 4 we show these current lines together with the magnetic field lines in a $3 \mathrm{D}$ volume rendering. The magnetic field lines we plot here are confined within the small cross-section of the actual loop seen in EUV and are the same field lines as shown in Fig. 2. These field lines within the EUV loop run more or less parallel without showing a notable sign of a helical structure. If we were to also plot field lines further away from the center of the EUV loop, these would run roughly parallel, too, also not showing clear signs of a helical structure (we did not show these field lines further out to avoid a cluttering of the figure). The two footpoints of the field lines of the EUV loop are rooted in the periphery of the magnetic centers, or sunspots, of the active region, as expected (we refer also to discussion and Fig. 9 in Chen et al. 2014).
In contrast to the magnetic field lines, the overall shape of the current lines is helical, winding around the magnetic field lines. To illustrate the overall structure of the current system, we highlight three types of current lines by color in Fig. 4 .

- The red current lines are traced starting from the positive magnetic polarity (right side of Fig. 4) and mostly continuously connect to the other negative magnetic polarity on the left side. They illustrate the continuous currents mostly antiparallel to the magnetic field. In addition, they show some winding around the magnetic field lines of the loop.

- In contrast, the purple current lines are traced from the negative magnetic pole on the left side and first closely follow the loop to the apex. However, following this they change their direction and connect back to the same negative magnetic polarity on the left side.

- The green current lines are traced from the apex of the loop in both directions towards the surface. These lines are helical and wind around the magnetic field lines of the loop. Furthermore, there seems to be more current lines connecting from the apex to the positive polarity than to the negative one.

These cases of current lines illustrate that the current along one single loop can have both signs, meaning that currents in the left leg of the loop are pointing downwards, so parallel to the magnetic field (cf. Fig. 3), and then further up change sign to become antiparallel with the magnetic field. This is because currents lines from outside the loop close inside the loop and thus run in the seemingly wrong direction. Only when considering the full $3 \mathrm{D}$ picture as in Fig. 4 can we understand the seemingly strange change of sign of the current within the loop as shown in Fig. 3 a. In their model, Archontis \& Hansteen (2014), investigate the injection of a magnetic flux sheet into a convection-driven model of the solar corona. They also find a complex current structure 
around a smooth magnetic field and in their model the currents are able to trigger several small flares.

Furthermore, the complex helical structure of the current lines in our work suggest that the force-freeness of the magnetic field is only partially fulfilled. This is true not only in the surroundings of the loop as illustrated by the current lines in Fig. 4, but also within the bright EUV loop, where in particular in the left quarter of the coronal part (from 0.1 to 0.3 normalized arc length) the angle between magnetic field and current is of the order of $30^{\circ}$, so certainly not (anti-)parallel.

\subsection{Driving at the base of the corona}

What drives the currents within the loops system to change sign? To answer this we investigate the currents and the driving forces at the base of the corona. The flip of sign of the currents with respect to the magnetic field (as seen in Fig. 3a) implies that on both sides the currents are downward directed. On the left footpoint the magnetic field is negative (see Fig. 5b), thus downward directed. There the angle $\gamma$ between magnetic field $\boldsymbol{B}$ and current is small (Fig. 2), so the currents are parallel to $\boldsymbol{B}$ and thus downwards. At the right footpoint the magnetic field is positive (upwards) but the angle $\gamma$ is about $180^{\circ}$, so the current is antiparallel to $\boldsymbol{B}$ and thus also downwards directed. This is also the case at the base of the corona. To illustrate this we show in Fig. 5a the vertical current at a constant height of $2.9 \mathrm{Mm}$. At the location where the field lines penetrate this height layer, the vertical currents at both loop footpoints are negative. That the downward directed currents at both footpoints are present at the base of the corona suggests that the direction of the currents in the loop is set by the dynamics at the bottom of the loop. An interesting feature is the bipolar curl structure surrounding the loop; in particular, it is significantly stronger than the overall current at this height (see Fig. 5a). Directly in the neighborhood of the loop footpoint, the currents are negative, whereas towards the center of the plane (away from the sunspot), the currents are positive. Because of the symmetry of the simulation, the bipolar vertical current structure near the two sunspots is symmetric (see Figs. 5a,b).

The currents at the base of the corona are generated by the velocity acting on the magnetic field. This can be seen by taking the curl of the induction equation, which describes the evolution of the current density. On the right-hand side (rhs), the dominant term is the double curl of the electromotive force $\boldsymbol{\nabla} \times \boldsymbol{\nabla} \times(\boldsymbol{u} \times \boldsymbol{B})$, whose vertical component is plotted in Fig. 5c. It shows clearly that the sign of current is generated by velocity field, which is driven by the convective motions in the photosphere that move and shear the magnetic field. However, the values indicate a typical timescale of just $10 \mathrm{~s}$ to produce the amount of current density seen in Fig. 5a.

Because of the small-scale flows in the photosphere and the resulting fluctuation of the (vertical) currents, it is difficult to pin-point the exact cause of the bipolar vertical current structure at the base of the corona (at a height of $2.9 \mathrm{Mm}$ as visualized in Fig. 5a). However, we see upflows into the loop and a coalescent flow driving the loop footpoints into the sunspots (as already pointed out by Chen et al. 2015). In accordance with the above discussion of the double-curled electromotive force (Fig. 5c), this flow structure is a good candidate for understanding the formation of the bipolar current structure at the loop footpoints.

In addition, we attempt to describe the current in the uncurled induction equation, where they are related to difference of (1) the electromotive force $\boldsymbol{u} \times \boldsymbol{B}$ and (2) the temporal evolution of the magnetic field or vector potential, respectively. The
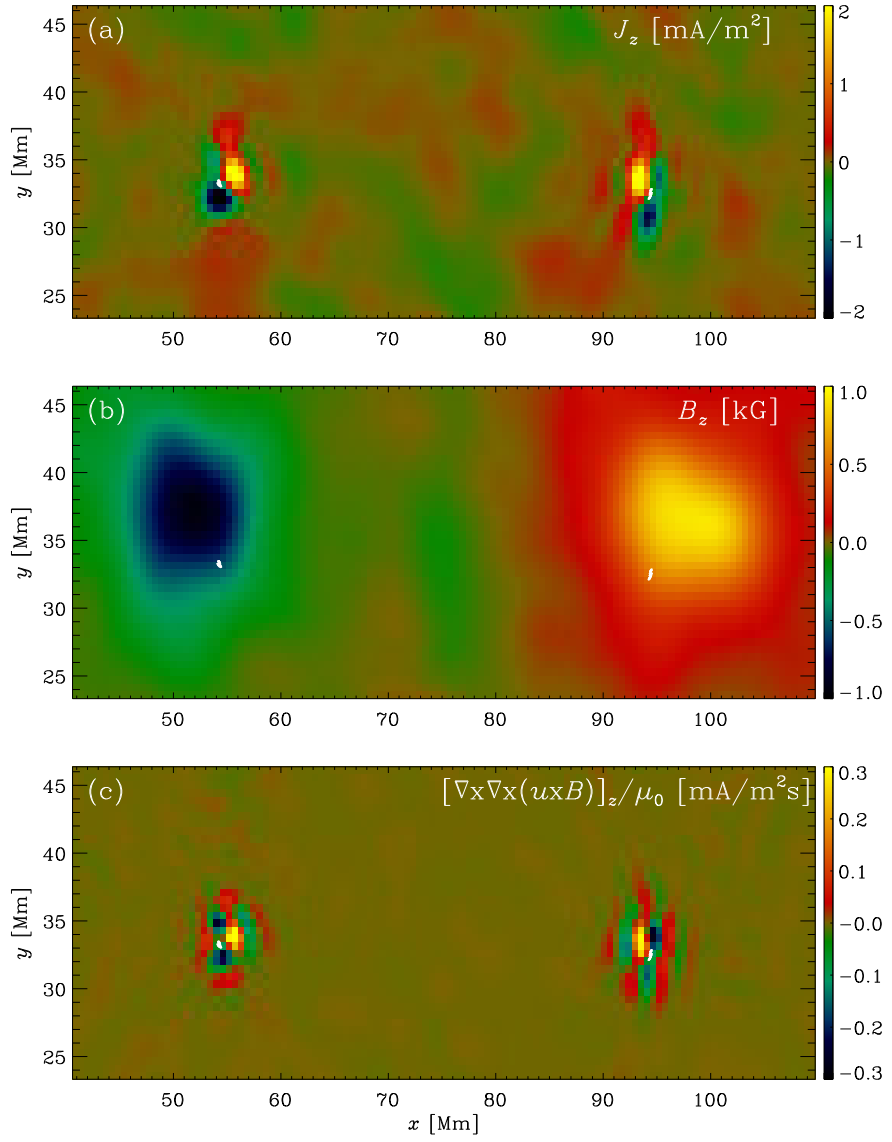

Fig. 5. Conditions at the base of the corona of Chen et al. (2014, 2015). Vertical current density $J_{z}$ (panel $a$ ), vertical magnetic field $B_{z}$ (panel $b$ ) and the vertical component of the double curl of the electromotive force $[\boldsymbol{\nabla} \times \boldsymbol{\nabla} \times(\boldsymbol{u} \times \boldsymbol{B})]_{z}$ (panel ) in a horizontal plane at a height of $z=2.9 \mathrm{Mm}$ placed around the field lines (white dots); we refer to Sect. 3.2.

second is governed by the magnetic field evolution enforced at the photospheric level, that is, the magnetic field in the form of flux tubes is pushed through the lower boundary. A detailed analysis reveals that from the photosphere to the base of the corona these two contributions, that is, the electromotive force and the time evolution, are similar in strength and structure resulting in a small difference, which is the curl of the current. A clear correlation between $\boldsymbol{u} \times \boldsymbol{B}$ and the vertical component of the current is not present, however. This is not surprising because the currents are the (small) difference of two larger quantities. The typical structure of the currents at the base of each loop footpoint seen at $z=2.9 \mathrm{Mm}$ in Fig. 5a is similar for heights near the coronal base. However, closer to the photosphere, the structure of the vertical currents becomes more complex, which is because of the small convection patterns. This renders the analysis of the ultimate origin of the sign of vertical current using the curled and uncurled induction equation in the photosphere an ill-posed problem.

The currents in the corona, and in the loop in particular, simply adjust to the current structure at the base of the corona. In particular, the bipolar current structure in Fig. 5a plays an important role in forming the current structure around and in the loop. To illustrate this fact, we show in Fig. 6 only a few selected magnetic field lines (gray) and current lines (red, yellow) connecting the bipolar vertical current concentration at the coronal base. One magnetic field line connects the positive (vertical) current on the left side to the positive current on the right side. 


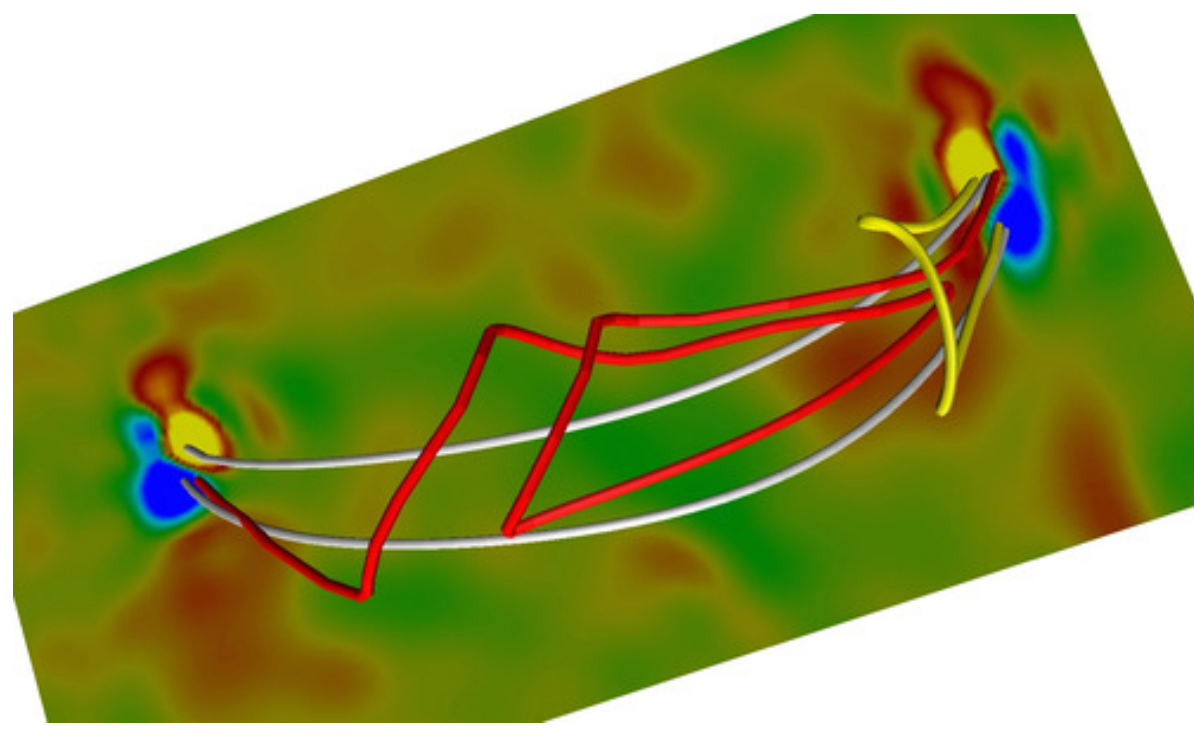

Fig. 6. 3D rendering of the current and magnetic field lines around the loop in a top view from above the active region of Chen et al. (2014, 2015). The colored plane at the bottom shows the vertical current $J_{z}$ at $z=2.9 \mathrm{Mm}$ at the base of the corona; the color-coding and scale are similar to those in Fig. 5a for the same quantity at the same height. The gray-colored lines display the magnetic field lines connecting the positive and negative vertical current concentrations with each other. The red line shows a current line (or a streamline of the vector field of the current) connecting the positive vertical current concentration on the right-hand side to the negative concentration on the left-hand side. The yellow line also shows a current line, but connecting the positive and negative current concentrations on the right-hand side. We refer to Sect. 3.2.

The other fieldline connects the two patches of negative currents. So, at each of the two footpoints of each fieldline, the vertical current points in the same vertical direction (both times in or out of the loop). This is not a feature of these two selected fieldlines, but a general feature and reflects the discussion of the currents along selected fieldlines in Sect. 3.1.

The current lines behave differently. One current line (red) starts from the positive concentration on the right-hand-side, close to one of the field lines and ends at the negative concentration of the left-hand-side close to other magnetic field lines. On its way from one side to the other it leaves the magnetic field line that it started with, winds around the magnetic field (indicating a circular component of the current) and ends up with the another magnetic field line. The other current line (yellow) starts on the right side not too far from the other current line at the positive concentration, but connects to the negative current concentration on the same right side close to the other field line. This clearly shows that the currents along magnetic field lines change their sign, because the current lines have to connect the two different current polarities and therefore cannot be aligned with the magnetic field. Furthermore, the bipolar current structure is crucial for the current field line to wind around the loop or even turn back to the same side of the loop. However, in Sect. 3.4 we discuss that the plasma flows within the loop also have an influence on the helical loop structure.

\subsection{Induced magnetic field and the coronal currents}

The complex helical current system around the bright loop structure is a consequence of the magnetic field structure and the forces associated with it. The large-scale current system in the corona associated with the loop turns out to be strongly influenced by induced coronal magnetic field that locally reduces the background magnetic field of the active region.

As a first step, we investigate how the magnetic field deviates from a current-free magnetic field, that is, a potential field. For this purpose, we extrapolate a potential field using the vertical magnetic field at the bottom boundary $(z=0)$. We identify the difference of the potential and the actual magnetic field as the induced magnetic field $\boldsymbol{B}^{\text {ind }}$. This is the component of the magnetic field associated with the currents. To illustrate the induced field we show a vertical cut through the mid-plane between the two loop footpoints $(y-z$ plane at $x=75 \mathrm{Mm})$ in Fig. 7a. Near
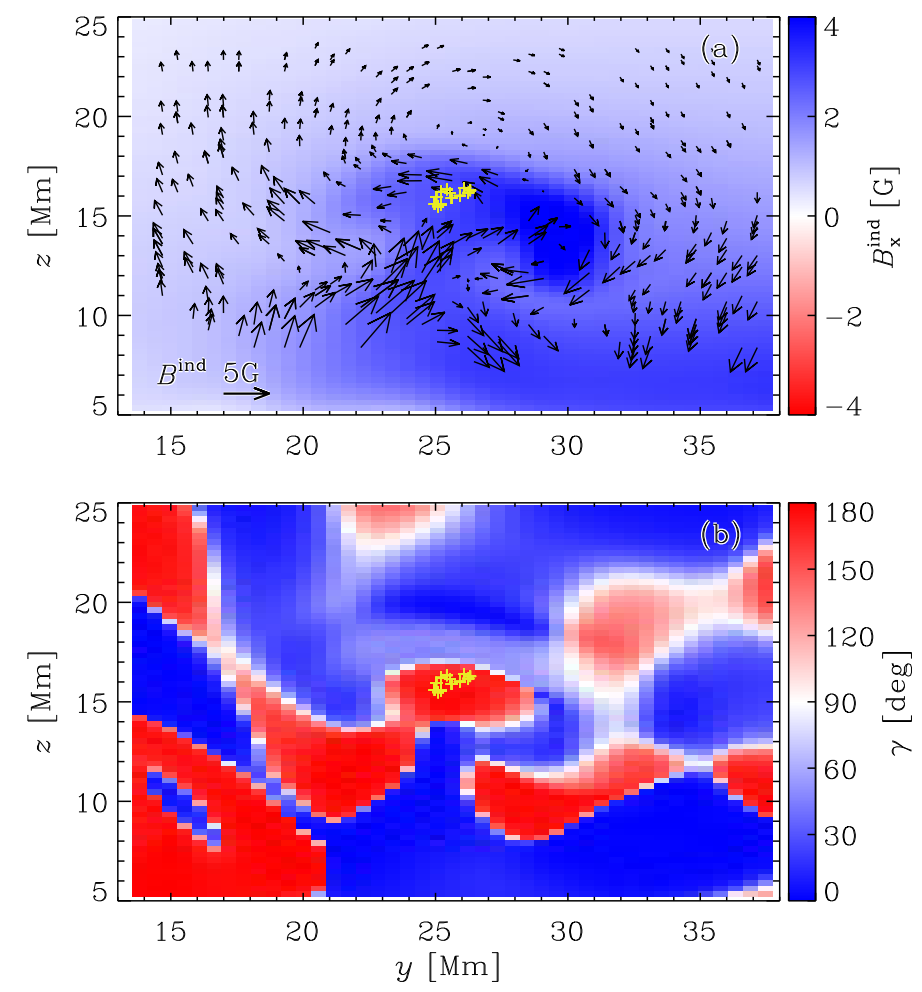

Fig. 7. Magnetic field strength and inclination at the apex of the loop at $x=75 \mathrm{Mm}$ of Chen et al. (2014, 2015). Panel $a$ : induced magnetic field: color-coded $B_{x}^{\text {ind }}$ together with magnetic field vectors $B^{\text {ind }}$ in $y$ $z$ plane. We note that the positive $x$ direction is pointing out of the plane of view. Panel $b$ : angle $\gamma$ between current density $\boldsymbol{J}$ and the magnetic field $\boldsymbol{B}$, see Eq. (2). The locations, where the magnetic field lines Fig. 3 breach through the plane are marked with yellow crosses. We refer to Sect. 3.3.

the loop apex (at $z \approx 15 \mathrm{Mm}$ ) $B_{x}^{\text {ind }}$ has up to $4 \mathrm{G}$ in the positive $x$ direction. Because the overall magnetic field $\boldsymbol{B}$ points in the negative $x$ direction at the apex with a strength of around $100 \mathrm{G}$, the induced field $\boldsymbol{B}^{\text {ind }}$ reduces the background magnetic field by around 5\%. The induced magnetic field in Fig. 7a has a shape similar to a tilted mushroom (also visible in Fig. 8 of Chen et al. 2014). The induced field vectors in the vertical cut form a lefthanded helical field above and beside the loop, where the field strength is smaller than in the $x$ direction (Fig. 7a). Below the 


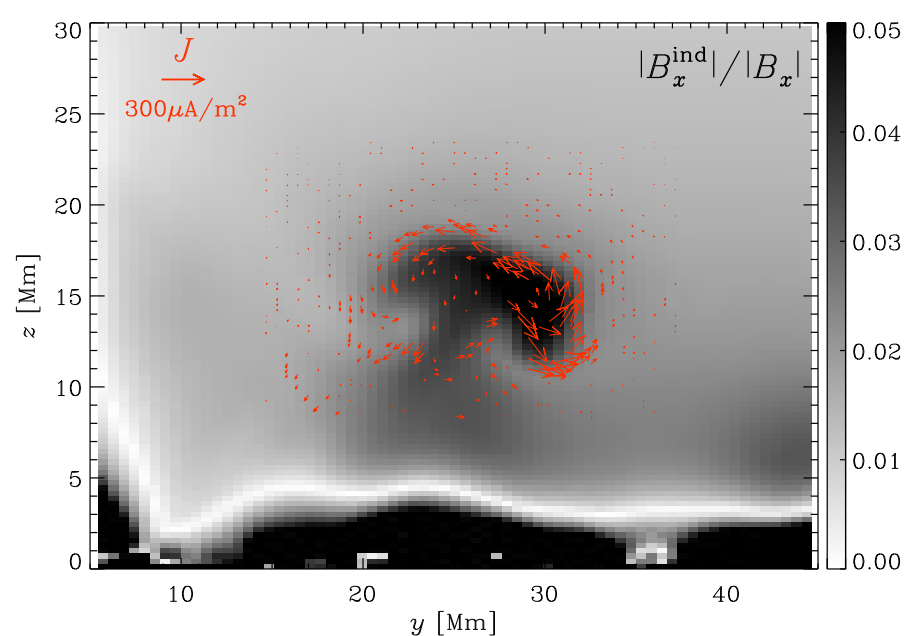

Fig. 8. Relative induced magnetic field and current density in the $y-z$ plane of the loop apex at $x=75 \mathrm{Mm}$ of Chen et al. $(2014,2015)$. The ratio of the modulus of the induced magnetic field in the $x$ direction $\left|B_{x}^{\text {ind }}\right|$ and the modulus of the total magnetic field $\left|B_{x}\right|$ is color-coded. The red arrows indicate vectors of the current density $\boldsymbol{J}$ in the $y$ - $z$ plane. We refer to Sect. 3.3.

loop, there exists a strong vertically upward-pointing induced magnetic field with a comparable strength to $B_{x}^{\text {ind }}$. In essence, while the overall magnetic field is running more or less straight from one footpoint to the other, this shows that the induced field has a clear helical structure. Nevertheless, because the induced field has a strength of only $5 \%$ of the overall background field, the helicity is not visible when simply plotting magnetic field lines.

To check that the induced magnetic field is consistent with the currents, we plot these together in Fig. 8. Here we see the mushroom-type enhancement of the induced field that is restricted to the vicinity of the loop. While the actual EUV loop visible in coronal emission has a diameter of some $2 \mathrm{Mm}$ (Chen et al. 2014), here the region of the induced magnetic field covers a region (in a vertical cut near the apex) of almost $10 \times 5 \mathrm{Mm}^{2}$. While in this region the induced field is of the order of $5 \%$ of the background field, it is practically zero outside in the corona (of course, in the photo- and chromosphere where the field is far from being force-free the induced magnetic field is much larger). The currents in the vertical cut in Fig. 8 show a clear counter-clockwise rotation, which according to the righthand-rule is directly related to induced magnetic field in the positive $x$ direction covering an area much larger than the EUV loop.

To relate the induced magnetic field to the current system around the loop, we define the angle $\gamma$ between current and magnetic field so that

$\cos \gamma=\frac{\boldsymbol{J} \cdot \boldsymbol{B}}{|\boldsymbol{J}||\boldsymbol{B}|}$

This allows us to quantify the discussion of the helical currents around the loop as visualized in Fig. 4. To this end we plot the angle $\gamma$ in Fig. $7 \mathrm{~b}$ in the same vertical mid-plane between the footpoints as in Fig. 7a. In accordance with Fig. 3 we see that at the position of the loop (indicated by the yellow crosses), the currents are antiparallel to the field $\left(\gamma \approx 180^{\circ}\right)$. In contrast, away from the center of the loop the magnetic field deviates significantly from a force-free state as emphasized by values of $\gamma$ close to $90^{\circ}$ with currents being almost perpendicular to the magnetic field, in particular in a large patch directly above the loop (see Fig. 7b). This consideration clarifies that the strongest deviation from a force-free field is found outside the loop. One might be tempted to conclude that the field is force-free inside the loop, where the EUV emission is strong. However, this would not be correct. While there the currents are indeed parallel or antiparallel to the field (consistent with force-free) the currents can switch sign in response to the closing of the current system surrounding the loop (cf. Sect. 3.1), which cannot be captured by a force-free description of the magnetic field.

We summarize that in a region significantly larger than the coronal loop seen in EUV we find a significant disturbance of the magnetic field directly related to the current systems. Inside and outside the EUV loop the assumption of a force-free field breaks down.

\subsection{Plasma flows and induced magnetic field}

To understand the origin of the induced magnetic field, we investigate the plasma motions in the loop structure. These motions can generate a (small) change in the magnetic field through the electromotive force. The plasma flows from the bottom of the corona into the loop are shown in Figs. 9a, b, where we plot the plasma velocities in the $y-z$ and $x-z$ planes, that is, in vertical planes across and along the loop. The flows are mostly upwards in both legs of the loop (Fig. 9b). The cut perpendicular to the loop in the $y-z$ plane reveals that the plasma moves upwards in the middle of the loop and disperses in the top of the loop in horizontal directions (Fig. 9a). Transported away from the loop the plasma falls down again generating a small vortex-like flow structure in the $y-z$ plane together with a velocity in the $x$ direction. In one of these vortex-like structures the $x$-component of the induced magnetic field has a maximum, indicated with the yellow contours in Fig. 9a. A vertical cut through this location at 29.5 Mm (Fig. 9b) shows the upflows along the legs of the loop, the horizontal flow at top of the loop, and the downflows below the apex. The upward motion along the legs is consistent with the analysis by Chen et al. (2014), where the flow is found to be driven by the Joule heating at the base of the corona.

The presence of the flow vortex and the induced magnetic field at the same location (Fig. 9a) indicates a connection between these two. To check this, we computed the curl of the electromotive force in the $x$ direction because this is the main contribution for changing $B_{x}$ in time. We find positive values for the electromotive force of some $20 \mathrm{mG} / \mathrm{s}$ to $60 \mathrm{mG} / \mathrm{s}$ (in the positive $x$ direction) at similar locations where also the large values of induced magnetic field are found (cf. Fig. 9c). In particular, this is also the case at the same height as the loop and slightly below. Analyzing the same simulation, Chen et al. (2014) found that the flows into the loop last some $100 \mathrm{~s}$ (their Fig. 3d). Consequently, together with the electromotive force, over this timescale we can expect an induced magnetic field of some $2 \mathrm{G}$ to $6 \mathrm{G}$. This is just the induced magnetic field we find (cf. Fig. 9d), that also points in the positive $x$ direction. This induced magnetic field then slightly suppresses the background magnetic field, which is pointing in the negative $x$ direction near the loop top. So from this we can conclude that the flow in the loop induces a magnetic field that is directed opposite to the background field and thus reduces the field strength in the loop.

The induced magnetic field also has components in the other directions (Fig. 9d), but we focus here on the $x$ component as it is tightly related to the helical currents around the loop; we refer to Sect. 3.3. However, the other components of the induced magnetic field can also be linked in a similar way to their corresponding components of the curl of electromotive force. 

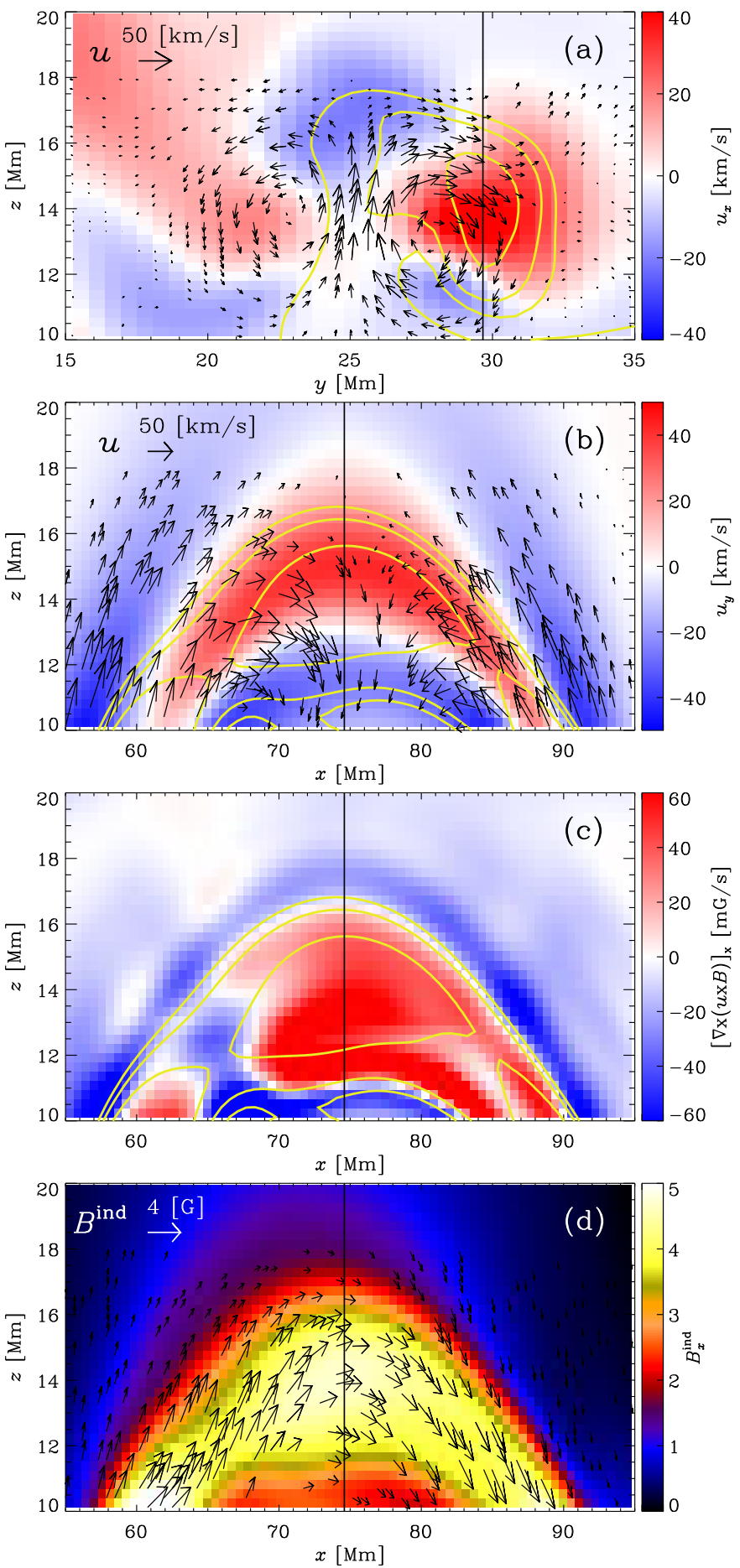

Fig. 9. Flows, curl of electromotive force, and induced magnetic field in the loop structure of Chen et al. $(2014,2015)$. The vertical cuts are perpendicular to the loop in the $y-z$ plane at $x \approx 75 \mathrm{Mm}$ (panel $a$ ) and along the loop in the $x-z$ plane at $y \approx 30 \mathrm{Mm}$ (panels $b-d$ ) as indicated by the vertical lines in the corresponding cuts. Panels $a, b$ show the velocity $\boldsymbol{u}$ in, the arrows indicating the components within the plane, and the color of the component out of the plane. Panel $c$ shows curl of the electromotive force in the $x$ direction $[\nabla \times(\boldsymbol{u} \times \boldsymbol{B})]_{x}$ color-coded in the $x-z$ plane. Panel $d$ displays the induced magnetic field $\boldsymbol{B}^{\text {ind }}$, color-coded for the $x$ component and the arrows showing the vectors in the $x-z$ plane. The yellow contours in panels $a-c$ indicate the levels of $2.5,3,4 \mathrm{G}$ for $B_{x}^{\text {ind }}$. We refer to Sect. 3.4.

As a next step we want to investigate which contribution of the flow acting on the magnetic field is dominant. The $x$

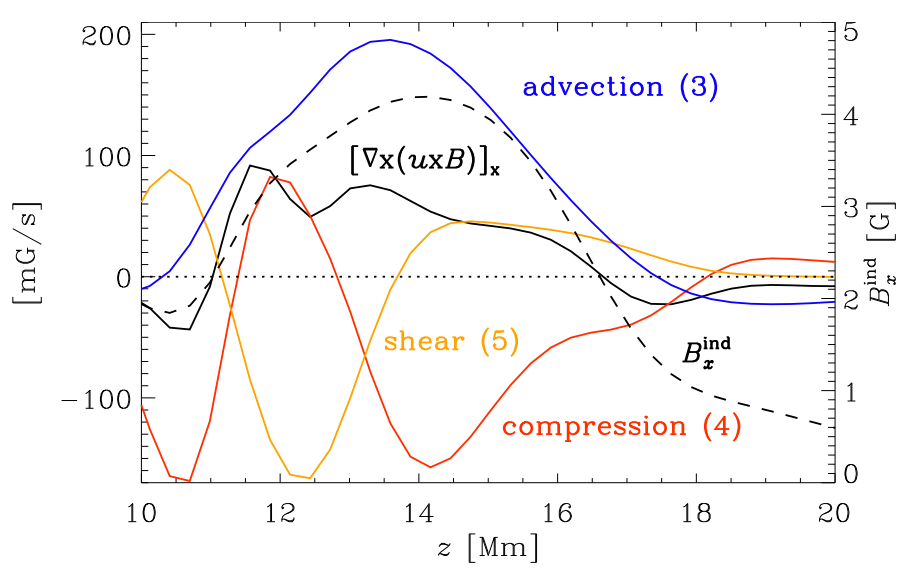

Fig. 10. $x$ component of the curl of electromotive force $[\boldsymbol{\nabla} \times(\boldsymbol{u} \times \boldsymbol{B})]_{x}$ (black solid line) together with its main contributions, advection (blue), compression (red) and shear (orange) plotted over height $z$ in the location of high induced magnetic field $(x=75 \mathrm{Mm}, y=30 \mathrm{Mm}$, also indicated in Figs. 9a, c as vertical lines); we refer also to Eqs. (3)-(6) for the contributions. Additionally we over-plot the same component of induced magnetic field $B_{x}^{\text {ind }}$ (dashed black). The horizontal black dotted line represents the zero value. Data from of Chen et al. $(2014,2015)$. We refer to Sect. 3.4.

component of curl of the electromotive force can be divided into four terms;

$[\boldsymbol{\nabla} \times(\boldsymbol{u} \times \boldsymbol{B})]_{x}=$

$-u_{y} \partial B_{x} / \partial y-u_{z} \partial B_{x} / \partial z \quad$ advection

$-B_{x} \partial u_{y} / \partial y-B_{x} \partial u_{z} / \partial z \quad$ compression

$+B_{y} \partial u_{x} / \partial y+B_{z} \partial u_{x} / \partial z$ shear

$+u_{x} \partial B_{y} / \partial y+u_{x} \partial B_{z} / \partial z$

solenoidality of $\boldsymbol{B}$.

We note that the terms $u_{x} \partial B_{x} / \partial x$ and $B_{x} \partial u_{x} / \partial x$ are often added for completeness, but because their contributions cancel out, we do not show them here. Furthermore, we prefer this kind of separation because it makes it easier to disentangle the different contributions. The last contribution turns out be small and is neglected in the following discussion. In Fig. 10, we plot the first three contributions over height at a location, where $B_{x}^{\text {ind }}$ is large in the middle of the loop ( $x=75 \mathrm{Mm}, y=30 \mathrm{Mm}$, also indicated in Figs. 9a, c as black vertical lines). Again, a positive electromotive force coincides with large values of $B_{x}^{\text {ind }}$. This is true between 11 and $17 \mathrm{Mm}$ in height. Below and above $[\boldsymbol{\nabla} \times(\boldsymbol{u} \times \boldsymbol{B})]_{x}$ is negative and $B_{x}^{\text {ind }}$ small. The positive values of the curl of electromotive force can be mostly associated with the advection (3) of magnetic field along the plasma. Between $z=14$ and 17 the contribution of the shear (5) and between $z=11$ and 13 the contribution of the compression (4) is also positive, but the advection (3) always dominates at these heights. A more detailed analysis reveals that compression becomes dominating in the legs. It is clear that considering all contributions is important to obtaining a full and accurate picture of magnetic field evolution. Pure advection would result in an overly large change in the magnetic field, therefore only fully compressible 3D MHD simulations are able to capture these plasma forces acting on the magnetic field and leading to the observed helical current structure.

The prominent flows and the induced magnetic field have an additional effect on the plasma and the force balance of the system. As shown in Fig. 8c of Chen et al. (2014), the loop shows a concentration of density in the top-right part of the mushroomtype structure seen in the cross-sectional cut of the loop. This 

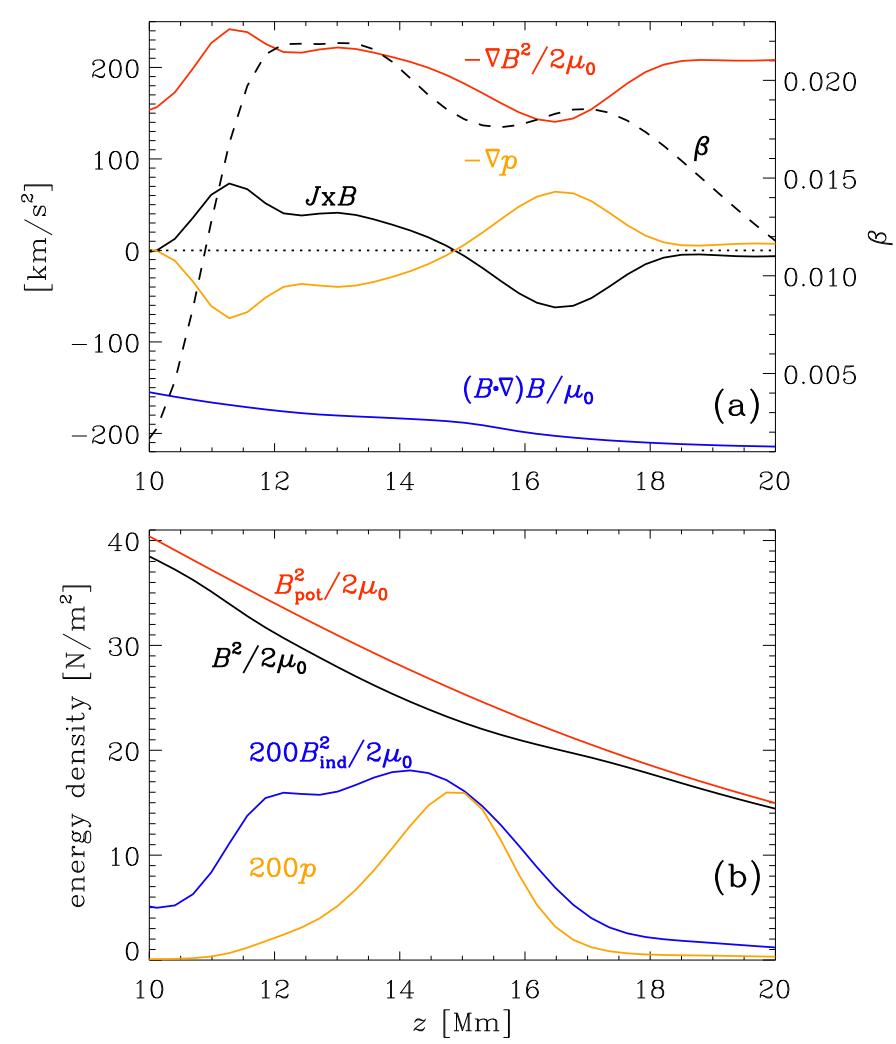

Fig. 11. Vertical force balance and energy density in the vicinity of the loop of Chen et al. (2014, 2015). Panel a: vertical force balance in terms of acceleration with contribution from the pressure gradient $\boldsymbol{\nabla} p$ (orange solid line) and the Lorentz force $\boldsymbol{J} \times \boldsymbol{B}$ (black) divided in the magnetic pressure gradient $\nabla B^{2} / 2 \mu_{0}$ (red) and the magnetic tension force $(\boldsymbol{B} \cdot \boldsymbol{\nabla}) \boldsymbol{B} / \mu_{0}$ (blue) plotted over height in the vicinity of the loop $(x=75 \mathrm{Mm}, y=30 \mathrm{Mm})$. We also show the plasma $\beta$ for the same region (black dashed) ( $y$-axis on right-hand side). Panel $b$ : energy densities of the total magnetic field $B^{2} / 2 \mu_{0}$ (black line), potential magnetic field $B_{\text {pot }}^{2} / 2 \mu_{0}$ and the induced magnetic field $B_{\text {ind }}^{2} / 2 \mu_{0}$ shown together with the thermal pressure $p$ (orange). The horizontal black dotted line in the upper plot represents the zero value. We refer to Sect. 3.4.

coincides well with the location of the induced magnetic field shown here in Figs. 7a and 9a. This local density enhancement can be explained by the upflows that transport material from the base of the corona into the loop structure. The density remains high there, because the magnetic field can keep it at the same position. The enhanced density leads to an enhanced pressure; the temperature is more uniformly distributed; we refer to Fig. 8a of Chen et al. (2014). In Fig. 11, we plot the force balance and the distribution of pressure and magnetic energy over height at the same location as in Fig. 10. The vertical force due to the pressure is exactly balanced by the magnetic pressure component of the Lorentz force (Fig. 11a). The induced magnetic field lowers the background magnetic field strength and therefore the magnetic pressure in this region. This allows the generation of a local enhancement of the gas pressure. As plotted in Fig. 11b, the gas pressure is enhanced in the same region, where the induced magnetic field is present. Even though the plasma $\beta$ is very small $(\beta \approx 0.018)^{3}$ in this region, the gradient of pressure balances the Lorentz force. This seems to be counter-intuitive. However, the

\footnotetext{
3 We note that the difference between the value of $\beta$ and the actual ratio of $p$ and $B^{2} / 2 \mu_{0}$ is due to the applied factor reducing the Lorentzforce, in the cases when the Alvfén speed becomes too large; we refer to Sect. 2 of Chen \& Peter (2015).
}

parts of the magnetic field and the pressure that do not change in space do not contribute to the force balance, but they define the value of $\beta$. Therefore, the value of plasma $\beta$ in this region is misleading when investigating the force balance that is governed by the gradients.

\subsection{Squashing of the loop}

The plasma flows into and through the loop structure have consequences for the evolution of the magnetic field. Chen et al. (2014) reported a squashing of the loop in the later evolution. They suggested, that the interaction of the magnetic field of the loop with overlying coronal magnetic field is responsible for compression and squashing of the loop. A detailed analysis reveals that this conclusion might not be correct. The flows shown in Figs. 9a, b act also on the magnetic field directly above the loop. As shown in the Fig. 9c, the resulting change in magnetic field through the electromotive force is negative above the loop structure and positive inside the loop. Even though this plot is a cut through the right part of the mushroom-type structure (cf. Fig. 8), the values are similar to those in the middle part of the loop. Because the background magnetic field points in the negative $x$ direction, outside (above) the loop the flow results in an amplification, inside the loop to a suppression of the magnetic field. The amplitudes of the resulting change in the magnetic field are around $50 \mathrm{mG} / \mathrm{s}$.

This change of the magnetic field induced by the flow in the loop might well account for the squashing of the magnetic structure reported by Chen et al. (2014). They found that the squashing of the loop takes place over the course of about 500 to $1000 \mathrm{~s}$. During this time the electromotive force (induced by the flow) of about $50 \mathrm{mG} / \mathrm{s}$ will change the magnetic field by some $25 \mathrm{G}$ to $50 \mathrm{G}$. This is a significant fraction of the background magnetic field strength, which is about $100 \mathrm{G}$ near the loop apex. Above the loop the background magnetic field gets significantly enhanced, within the loop reduced. Essentially this looks like a pile-up of magnetic field above the expanding loop, or like a squashing of the loop.

We did not find any evidence that the overlying magnetic field plays an important role in the squashing process. Its contribution would show up in the diffusion term of the induction equation, but this term is one order of magnitude smaller than the flow acting on the magnetic field. Therefore we conclude that the expanding loop gets squashed through a change of magnetic field induced by the plasma flow through the loop.

\section{Conclusions}

In this work we analyzed the magnetic field and current structure of a bright coronal loop of the three-dimensional MHD simulation of Chen et al. (2014). We found that while the magnetic field lines follow the loop structure as a concentrated flux bundle, the current stream lines form a complex structure. In particular, in the coronal part of the loop the current density undergoes a change of direction along the loop. This behavior is related to the footpoint motions in the photosphere, where a bipolar vertical current structure is generated. Because the magnetic field connects smoothly the current concentrations with the same vertical direction, the currents cannot be aligned with the magnetic field. Furthermore, the bipolar current structures play an important role in forming a complex current system because the current stream lines have to wind around the loop to connect to the bipolar current concentration on the other side of the loop. 
Most of the coronal part of the simulation is close to a current-free magnetic field configuration. We find that the currents are directly related to a reduction of the background magnetic field by about $4 \%$, forming an isolated non force-free structure. Because this structure has the same location as the hot bright coronal loop, a non-force-free state seems to be a necessity for heating the loop to coronal temperatures.

The local reduction of the background magnetic field can be explained by plasma flows into and through the loop acting on the magnetic field. In agreement with Chen et al. (2014), we found upward flows along the legs. The upward directed expansion of the loop creates vertical vortices next to the loop. This flow structure can be directly linked to the reduction of the magnetic field due to the curl of the electromotive force. The main contribution is the advection of field, but also compression and shear play a role.

The plasma flows acting on the magnetic field reduce the magnetic pressure. This allows for the stable concentration of density inside the loop, because the pressure gradient is balanced by the Lorentz force. This enhancement of density is crucial for the emission from the loop seen in extreme UV wavelengths. Additionally, we can relate the flow structure to the magnetic field evolution and explain the squashing of the loop at later times.

This work shows that a realistic modeling of a coronal loop structure, which produces realistic emissivity in coronal lines, needs to resolve the three dimensional structure of complex magnetic field and current systems. Even in a low- $\beta$ environment, the plasma properties, that is, flow, density, and pressure, will significantly influence the magnetic field structure and evolution. This is in particular true if the coronal loop is formed above an emerging active region. Therefore, one dimensional loop models (e.g., Klimchuk 2006), force-free extrapolations (Wiegelmann 2008) and magneto-frictional models (e.g., Cheung \& DeRosa 2012) are most likely not able to reproduce the complex helical current structure and the related UV emission as found in our models.

The model analyzed here, of course, is not a perfect representation of an emerging active region on the Sun. The coronal model discussed here builds on a model for the emergence of magnetic flux from the upper convection zone to the surface (Rempel \& Cheung 2014). On one hand, the flux emergence in this model, as well as in the related model of Cheung et al. (2010) is significantly faster than in the real Sun (Birch et al. 2016). Therefore, the changes in our modeled coronal magnetic field might be faster than on the Sun. On the other hand, the flux tube emerging in our model is not twisted (in contrast to the model of Cheung et al. 2010). A flux tube with a significant twist breaking through the surface would lead to a significant amount of magnetic helicity being injected into the corona and thus could lead to highly twisted magnetic structures in the corona. In that sense the considerations presented here represent lower limits for the amount of complexity of the current structures in and around coronal loops.

Further studies will involve the investigation of key parameters responsible for complex current systems. One possibility could be indeed the magnetic helicity, which is known to play an important role in the formation of unstable loop structures (e.g., Török \& Kliem 2005; Pariat et al. 2015) and is connected to the underlying dynamo (e.g., Warnecke \& Brandenburg 2010; Warnecke et al. 2011, 2012). Another future work path would be to perform realistic coronal simulations on top of selfconsistently formed bipolar flux concentration, either in convection (Stein \& Nordlund 2012; Käpylä et al. 2016) or forced turbulence (Warnecke et al. 2013, 2016).

Acknowledgements. The authors thank the anonymous referee and Matthias Rempel for useful comments and suggestions. The simulations have been carried out on supercomputers at Gesellschaft für wissenschaftliche Datenverarbeitung mbH Göttingen (GWDG), and on the supercomputers at Max Planck Computing and Data Facility (MPCDF) in Garching and SuperMUC. We thank the Partnership for Advanced Computing in Europe (PRACE) for awarding us the access to SuperMUC based in Germany at the Leibniz Supercomputing Center (LRZ). J.W. acknowledge funding by the Max-Planck/Princeton Center for Plasma Physics and the funding from the People Programme (Marie Curie Actions) of the European Union's Seventh Framework Programme (FP7/20072013) under REA grant agreement No. 623609. This work was partially supported by the International Max-Planck Research School (IMPRS) on Physical Processes in the Solar System and Beyond (F.C.). The National Center for Atmospheric Research is sponsored by the National Science Foundation. The visualizations of Figs. 4 and 6 were done using VAPOR (www . vapor . ucar . edu).

\section{References}

Archontis, V., \& Hansteen, V. 2014, ApJ, 788, L2

Bingert, S. 2009, Ph.D. Thesis, Albert-Ludwig-Universität Freiburg Bingert, S., \& Peter, H. 2011, A\&A, 530, A112

Bingert, S., \& Peter, H. 2013, A\&A, 550, A30

Birch, A. C., Schunker, H., Braun, D. C., et al. 2016, Sci. Adv., 2, e1600557

Boerner, P., Edwards, C., Lemen, J., et al. 2012, Sol. Phys., 275, 41

Bourdin, P.-A., Bingert, S., \& Peter, H. 2013, A\&A, 555, A123

Chen, F., \& Peter, H. 2015, A\&A, 581, A137

Chen, F., Peter, H., Bingert, S., \& Cheung, M. C. M. 2014, A\&A, 564, A12

Chen, F., Peter, H., Bingert, S., \& Cheung, M. C. M. 2015, Nat. Phys., 11, 492

Cheung, M. C. M., \& DeRosa, M. L. 2012, ApJ, 757, 147

Cheung, M. C. M., Rempel, M., Title, A. M., \& Schüssler, M. 2010, ApJ, 720, 233

Galsgaard, K., \& Nordlund, A. 1996, J. Geophys. Res., 101, 13445

Gudiksen, B. V., \& Nordlund, Å. 2002, ApJ, 572, L113

Gudiksen, B. V., \& Nordlund, A. 2005, ApJ, 618, 1020

Hansteen, V. H., Hara, H., De Pontieu, B., \& Carlsson, M. 2010, ApJ, 718, 1070

Käpylä, P. J., Brandenburg, A., Kleeorin, N., Käpylä, M. J., \& Rogachevskii, I. 2016, A\&A, 588, A150

Klimchuk, J. A. 2006, Sol. Phys., 234, 41

Mok, Y., Mikić, Z., Lionello, R., \& Linker, J. A. 2005, ApJ, 621, 1098 Mok, Y., Mikić, Z., Lionello, R., \& Linker, J. A. 2008, ApJ, 679, L161 Myers, C. E., Yamada, M., Ji, H., et al. 2015, Nature, 528, 526

Oz, E., Myers, C. E., Yamada, M., et al. 2011, Phys. Plasmas, 18, 102107 Pariat, E., Valori, G., Démoulin, P., \& Dalmasse, K. 2015, A\&A, 580, A128 Parker, E. N. 1972, ApJ, 174, 499

Peter, H. 2015, Phil. Trans. R. Soc. London Ser. A, 373, 20150055

Peter, H., Gudiksen, B. V., \& Nordlund, A. 2004, ApJ, 617, L85

Peter, H., Gudiksen, B. V., \& Nordlund, A. 2006, ApJ, 638, 1086

Peter, H., Warnecke, J., Chitta, L. P., \& Cameron, R. H. 2015, A\&A, 584, A68 Rappazzo, A. F., Velli, M., Einaudi, G., \& Dahlburg, R. B. 2008, ApJ, 677, 1348 Rempel, M., \& Cheung, M. C. M. 2014, ApJ, 785, 90

Stein, R. F., \& Nordlund, A.. 2012, ApJ, 753, L13

Török, T., \& Kliem, B. 2005, ApJ, 630, L97

Warnecke, J., \& Brandenburg, A. 2010, A\&A, 523, A19

Warnecke, J., Brandenburg, A., \& Mitra, D. 2011, A\&A, 534, A11

Warnecke, J., Käpylä, P. J., Mantere, M. J., \& Brandenburg, A. 2012, Sol. Phys., 280, 299

Warnecke, J., Losada, I. R., Brandenburg, A., Kleeorin, N., \& Rogachevskii, I. 2013, ApJ, 777, L37

Warnecke, J., Losada, I. R., Brandenburg, A., Kleeorin, N., \& Rogachevskii, I. 2016, A\&A, 589, A125

Wiegelmann, T. 2008, J. Geophys. Res., 113, A12 